\title{
Intraretinal Transplantation for Rod-Cell Replacement in Light-Damaged Retinas*
}

\author{
Manuel del Cerro, Mary F.D. Notter, Coca del Cerro, \\ Stanley J. Wiegand, Donald A. Grover, and Eliot Lazar \\ Departments of Neurobiology, Anatomy and Ophthalmology, \\ University of Rochester Medical School \\ Rochester, New York, USA
}

\begin{abstract}
Blindness from retinal disease is often the consequence of extensive damage to the photoreceptor cell population, while other cell types which form the neural retina are relatively spared. In this setting, transplantation of photoreceptor cells could offer hope for the restoration of some degree of visual function. We tcsted the feasibility of this approach by transplanting immature retinal cells into the eyes of adult rats affected by late stage phototoxic retinopathy, which are almost totally devoid of photoreceptor cells.

Dissociated neuroretinal cells from newborn rats were injected into the hosts' retinas. These cells were labelled with the fluorescent tracer Fast-blue for identification within the host eye. Survival time ranged from 3 to 100 post-transplantation days.

Fundus examination of light-irradiated eyes showed pallor caused by a considerable reduction of the retino-choroidal vascular bed after light irradiation. Histologically the hosts exhibited decimation of the elements forming the outer layers throughout the entire retina.

As visualized by light and electron microscopic procedures, we report the differentiation of clusters of transplanted photoreceptor cells, and the integration of these cells within the adjacent areas of the host retina.
\end{abstract}

Reprint address:

Manuel del Cerro

Departments of Neurobiology,

Anatomy and Ophthalmology

University of Rochester Medical School

Rochester, New York, USA
Fluorescence microscopy showed these clusters to be formed by fluorescently labelled cells developing in intimate contact with the unlabelled host retina. Electron microscopically it was possible to determine that these photoreceptors had established synaptic contacts. These observations indicate that successful transplantation of immature retinal cells is feasible into adult eyes that have suffered extensive retino-choroidal damage. These findings also support the concept that retinal transplantation is a procedure which may open new avenues into the study of retinal repair.

\section{INTRODUCTION}

Grafting of immature retinal cells is one of the more novel approaches applied to the study of retinal repair and regeneration. The dramatic advances in neural transplantation made over the course of this decade have raised hopes that intraocular retinal transplants may be an effective means of providing viable cells to an irreversibly injured adult retina. At the very least, retinal transplants may shed new light on aspects of retinal development and plasticity; while at best they could provide the means for repairing damaged retinas. Recently we have reported on retinal transplants carried out under a variety of conditions, including across immunologic barriers $/ 9,10,11,12 /$. Following that sequence of studies, this paper describes the results obtained from transplanting cell suspensions of perinatal retina into adult hosts affected by late stage phototoxic retinopathy. The results of these experiments are compared with the findings of previous studies dealing with retinal transplantation in the eye and central nervous tissue of mammalian species.

* Early results from this study have been communicated to the Schmitt Symposium on Transplantation into the Mammalian CNS, and to the Symposium on Inherited and Environmentally Induced Retinal Degenerations (del Cerro et al., 1988b, and del Cerro et al., 1989). 


\title{
Intraretinal Transplantation for Rod-Cell Replacement in Light-Damaged Retinas*
}

\author{
Manuel del Cerro, Mary F.D. Notter, Coca del Cerro, \\ Stanley J. Wiegand, Donald A. Grover, and Eliot Lazar \\ Departments of Neurobiology, Anatomy and Ophthalmology, \\ University of Rochester Medical School \\ Rochester, New York, USA
}

\begin{abstract}
Blindness from retinal disease is often the consequence of extensive damage to the photoreceptor cell population, while other cell types which form the neural retina are relatively spared. In this setting, transplantation of photoreceptor cells could offer hope for the restoration of some degree of visual function. We tcsted the feasibility of this approach by transplanting immature retinal cells into the eyes of adult rats affected by late stage phototoxic retinopathy, which are almost totally devoid of photoreceptor cells.

Dissociated neuroretinal cells from newborn rats were injected into the hosts' retinas. These cells were labelled with the fluorescent tracer Fast-blue for identification within the host eye. Survival time ranged from 3 to 100 post-transplantation days.

Fundus examination of light-irradiated eyes showed pallor caused by a considerable reduction of the retino-choroidal vascular bed after light irradiation. Histologically the hosts exhibited decimation of the elements forming the outer layers throughout the entire retina.

As visualized by light and electron microscopic procedures, we report the differentiation of clusters of transplanted photoreceptor cells, and the integration of these cells within the adjacent areas of the host retina.
\end{abstract}

Reprint address:

Manuel del Cerro

Departments of Neurobiology,

Anatomy and Ophthalmology

University of Rochester Medical School

Rochester, New York, USA
Fluorescence microscopy showed these clusters to be formed by fluorescently labelled cells developing in intimate contact with the unlabelled host retina. Electron microscopically it was possible to determine that these photoreceptors had established synaptic contacts. These observations indicate that successful transplantation of immature retinal cells is feasible into adult eyes that have suffered extensive retino-choroidal damage. These findings also support the concept that retinal transplantation is a procedure which may open new avenues into the study of retinal repair.

\section{INTRODUCTION}

Grafting of immature retinal cells is one of the more novel approaches applied to the study of retinal repair and regeneration. The dramatic advances in neural transplantation made over the course of this decade have raised hopes that intraocular retinal transplants may be an effective means of providing viable cells to an irreversibly injured adult retina. At the very least, retinal transplants may shed new light on aspects of retinal development and plasticity; while at best they could provide the means for repairing damaged retinas. Recently we have reported on retinal transplants carried out under a variety of conditions, including across immunologic barriers $/ 9,10,11,12 /$. Following that sequence of studies, this paper describes the results obtained from transplanting cell suspensions of perinatal retina into adult hosts affected by late stage phototoxic retinopathy. The results of these experiments are compared with the findings of previous studies dealing with retinal transplantation in the eye and central nervous tissue of mammalian species.

* Early results from this study have been communicated to the Schmitt Symposium on Transplantation into the Mammalian CNS, and to the Symposium on Inherited and Environmentally Induced Retinal Degenerations (del Cerro et al., 1988b, and del Cerro et al., 1989). 
optics (Leitz Z "filter block" combination: excitation filter $335-425 \mathrm{~nm}$, barrier filter $460 \mathrm{~nm}$ ) to verify label incorporation as well as the absence of Fast Blue crystals in the medium. Labelled cells were maintained in CMF glucose medium at $4^{\circ} \mathrm{C}$ until transplantation. Viable transplants were obtained with cell suspensions that were kept on ice for as long as three hours, the longest time tested.

\section{Transplantation Procedure}

Dissociated cells, labelled and adjusted to the final concentration of $600,000 / \mu 1$, were injected into the eyeball of anesthetized hosts. The injection was made using a 27 gauge needle fitted to a microliter syringe containing the cell suspension and kept on crushed ice until the moment of use. The injection point was located at the 12 o'clock position, behind the eye equator. This point was exposed by gently rotating the eyeball downwards with the help of a fine toothed forcepts. A two $\mu$ injection of suspended cells with given over a period of approximatley 2 min. Using a Hamilton microsyringe, the cells were delivered at a point just behind the eye equator. The penetration of the needle was limited to approximately $1 \mathrm{~mm}$, in order to favor the release of the cells within the thickness of the host retina.

\section{Post-Transplantation Survival and Clinical Studies}

Survival times ranged from 3 to 100 days post-transplantation. The animals received repeated fundoscopic examinations during this time. For this purpose the animals were lightly anesthetized with intramuscular ketamine and xylazine and examined before and after the application of dilating drops. Features of interest were photographed using a Kowa RC-2 fundus camera in conjunction with a 20 diopters aspheric lens.

Animal handling was performed in compliance with NIH policies for humane treatment of research animals.

\section{Histological Techniques}

At the end of the survival period, the animals were again anesthetized with ketamine and xylazine, and perfused through the heart with a mixture of $2 \%$ glutaraldehyde and $2 \%$ paraformaldehyde in a $0.1 \mathrm{M}$ cacodylate buffer, ( $\mathrm{pH}$ 7.2-7.4). One hour after starting the perfusion, the eyes were enucleated, hemisected, and kept overnight at $4^{\circ} \mathrm{C}$ under fresh fixative. At this point the nasal and temporal halves of the hemisected eyes were observed under an Olympus SZH stereo microscope. After post-fixation in a chromate-osmium tetroxide solution /6/ and embedding in Eponate 12 (Ted Pella, Redding, CA), $1 \mu \mathrm{m}$ thick sections of the tissue were cut and stained with Stevenel Blue /7,8/ for light microscopy study. Ultrathin sections were cut with a diamond knife for electron microscopical studies; they were "stained" with lead acetate $/ 22 /$ and studied under a Zeiss 10 electron microscope operating at $80 \mathrm{kv}$.

\section{Quantitative Determinations}

Using a calibrated ocular reticle, the retinal thickness and the numbers of photoreceptor nuclei were measured in $1 \mu \mathrm{m}$-thick saggital sections of the eye. For this purpose the retina was divided into six sectors of equal length, designated as superior peripheral, equatorial, and central, and inferior central, equatorial and peripheral, respectively. For each section, three measurements were made on each of the six sectors, and the results from each sector were averaged.

\section{RESULTS}

\section{In Vivo Observations}

The normal fundus of the young Lewis rat (Fig. 1) presents a centrally located, relatively small round optic nerve head. Six to eight arteries and veins radiate from the head of the optic nerve in a spoke-like fashion. The veins alternate with the arteries and are one and a half to two times as wide as the arteries. As the vessels leave the optic nerve head they tend to protrude directly forward, then gradually slope downwards toward the periphery. These vessels tend to have a straight course as they extend towards the retinal periphery, but send out multiple arborizations. The far retinal periphery is difficult to observe because of the astigmatism induced by the large round lens, characteristic of the rat eye. The optic nerve varies in color from red, to orange or pink. In the absence of pigment, the large choroidal vessels, which take long sinuous courses, can be easily observed. Fundoscopic observation of the same animals after a period of continuous light exposure shows thinning of the retina, and a concomitantly severe attenuation of the vascular beds of the retina and choroid (Fig. 2). When examination of grafted animals was undertaken it was possible in some cases to confirm the survival and growth of the graft and to observe the focal rearrangement of vascularity through the transparent media of the recipient eye (Fig. 3). 
Histological Effects of Light-Irradiation on the Retina and Choroid

Histologically, the retina of animals exposed to continuous fluorescent illumination differs dramatically from that of sex and age-matched controls (Figs. 4,5). Quantitative observations underlined the severity and extent of the damage. They indicated that the total retinal thickness was greatly reduced in the peripheral, equatorial, and central retinal sectors, both in the superior and inferior hemispheres (Table 1). More significantly from a functional viewpoint, the number of rod cell nuclei seen in $1 \mu \mathrm{m}$-thick sections was reduced
TABLE 1

RETINAL THICKNESS

A) Thickness of the Normal Retina $(\mathrm{N}=6)$

\begin{tabular}{lrrrrrr} 
& \multicolumn{1}{l}{ SP } & \multicolumn{1}{l}{ SE } & \multicolumn{1}{l}{ SC } & \multicolumn{1}{l}{ IC } & \multicolumn{1}{l}{ IE } & \multicolumn{1}{l}{ IP } \\
Av. & 121.1 & 180.8 & 193.1 & 187.0 & 178.6 & 137.1 \\
S.D. & 3.2 & 3.9 & 5.8 & 7.6 & 5.4 & 3.6
\end{tabular}

B) Thickness of the Light-Damaged Retina $(\mathrm{N}=6)$

\begin{tabular}{rrrrrrr} 
& \multicolumn{1}{c}{ SP } & SE & \multicolumn{1}{l}{ SC } & \multicolumn{1}{l}{ IC } & \multicolumn{1}{c}{ IE } & \multicolumn{1}{l}{ IP } \\
Av. & 64.4 & 85.7 & 109.2 & 111.2 & 99.1 & 94.8 \\
S.D. & 11.1 & 7.6 & 9.4 & 5.0 & 9.9 & 9.8 \\
\hline
\end{tabular}

$\mathrm{SP}, \mathrm{SE}$ and SC indicate superior periphral, equatorial and central retinal locations. IC, IE and IP indicate irferior central, equatorial and peripheral retinal locations.

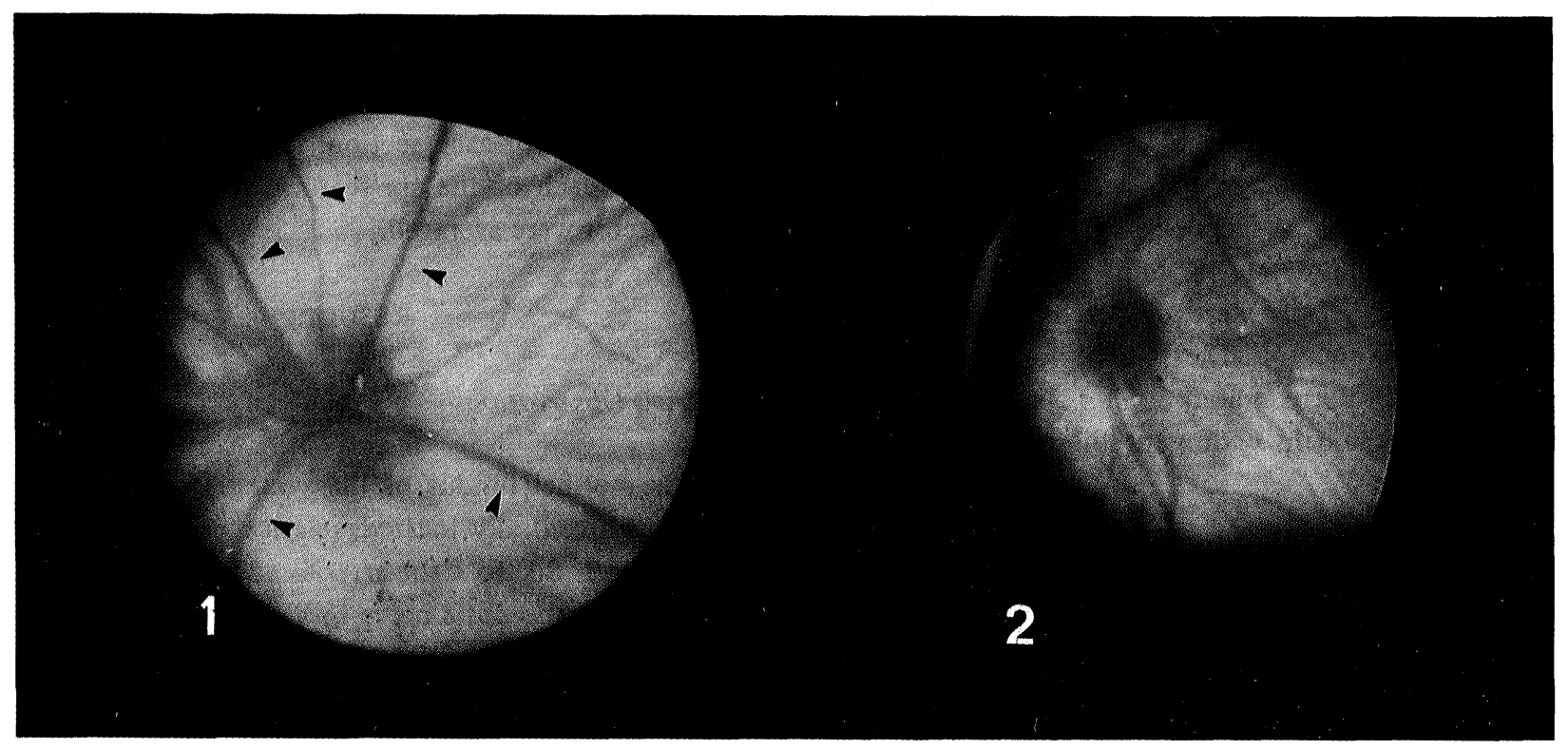

Fig. 1: Fundoscopic view of a normal two months old Fisher 344 albino rat. Retinal arteries and veins (arrowheads) radiate from the head of the optic nerve following a straight course towards the periphery. In the absence of pigment the large choroidal vessels, which take long sinuous courses, can be observed.

Fig. 2: Fundoscopic picture of the same eye after five weeks of continuous light irradiation. The retinal vessels are dramatically reduced in the caliber and follow a less regular course. The concomitant reduction in the retinal capillary bed permits an enhanced view of the choroidal vasculature.

Fig. 3: High magnification fundoscopic view of a retinal sector with a transplant growing in it. The site had an increased vascular supply as compared with other areas of the same retina. Microscopic sections of this eye confirmed the presence of transplanted cells in this location. Light-damaged host, 10 days after transplantation.

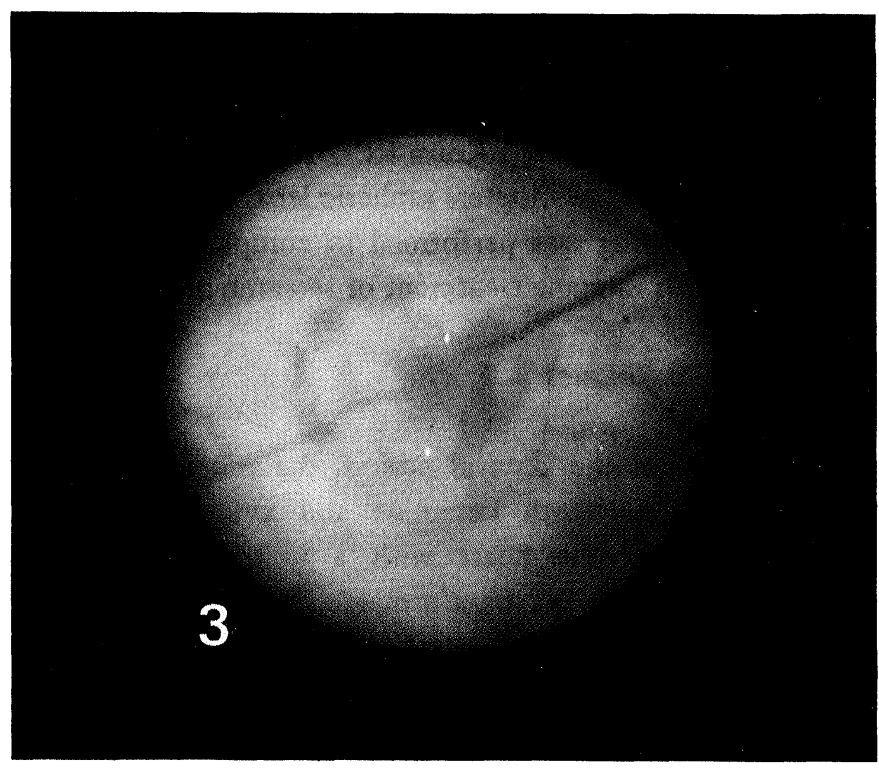




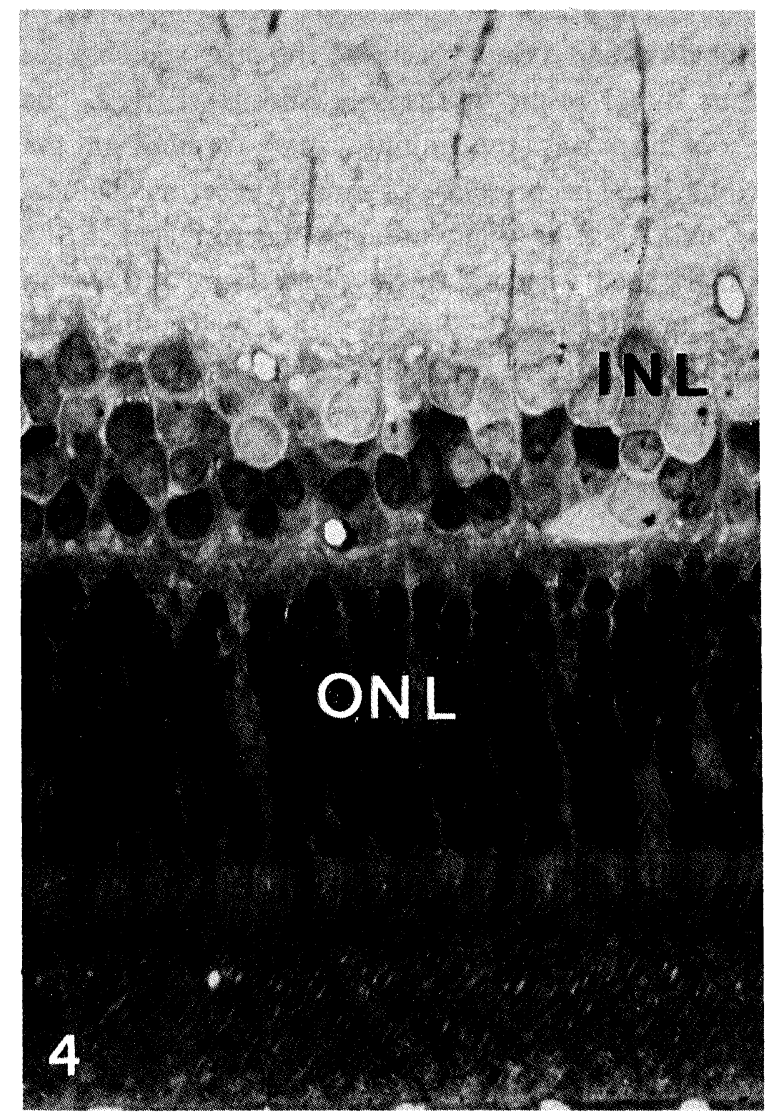

Fig. 4: Normal retina, from a control animal. The inner and outer nuclear layers are indicated (INL, ONL). Two $\mu \mathrm{m}$-thick plastic section. Magnification $375 \mathrm{x}$.

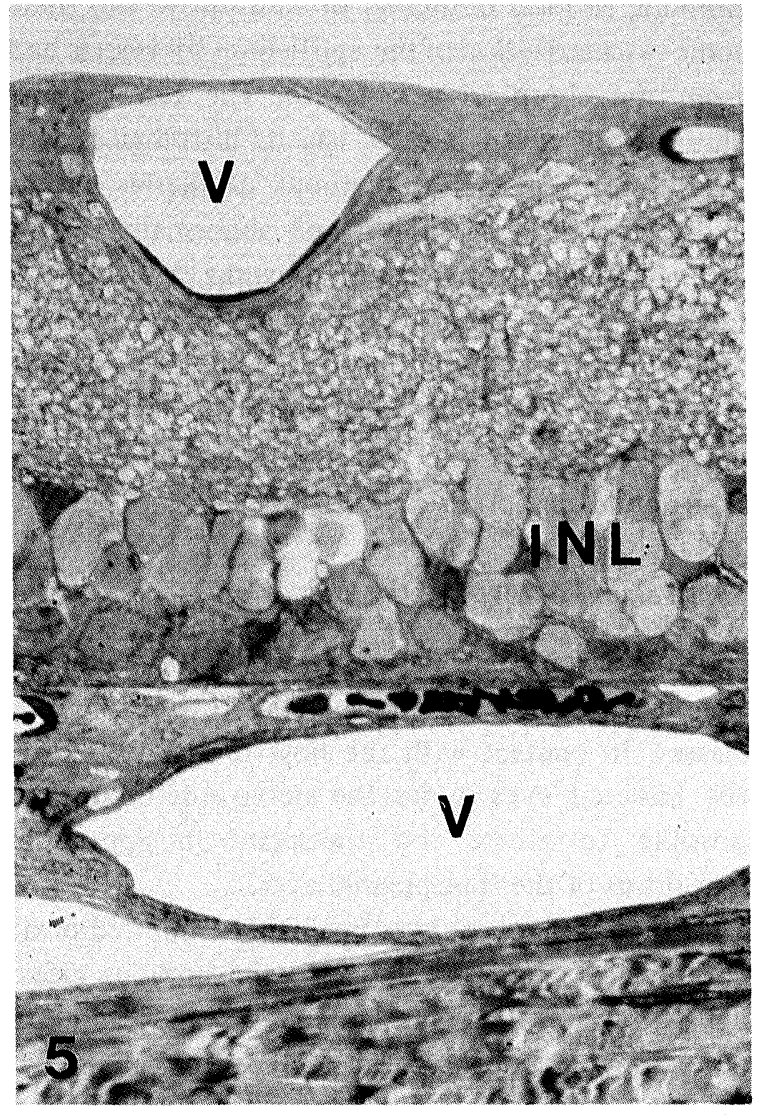

Fig. 5: Microphotograph of a light-damaged retina. All of the outer retinal layers have degenerated, as a result the inner nuclear layer (INL) contacts the retinal pigment epithelium. The capillary bed is reduced; dilated blood vessels (V) appear in the retina and choroid. Two $\mu \mathrm{m}$-thick plastic section. Magnification $375 \mathrm{x}$.

elements appeared hypertrophic, and partially filled the void left by the degenerated photoreceptors. In some instances the number of nuclear profiles seen in the inner nuclear layer was lower than present in the retinas of normal rats of the same strain. The retinal pigment epithelium exhibited focal changes ranging from focal

TABLE 2

ROD NUCLEAR PROFILES/250/MM OF SUPERIOR EQUATORIAL RETINA

row in the retinal periphery. Not only were these cells highly abnormal, but as shown in Table 2 , their numbers represented only a very small fraction of the normal population. The apical cytoplasmic processes of the Muller cells formed now the outer surface of the neural retina. They intermingled extensively with the microvilii emerging from the apical surface of the RPE. Both to the point of near extinction, particularly in the Qualitatively the changes were equally dramatic. Light and electron microscopical observations confirmed the virtually complete degeneration of elements forming complete absence of photoreceptor inner and outer segments throughout the retina. A few dystrophic rod cells consisting of a small spherical soma and a rudimentary pedicle were found forming a single, discontinuous 
thinning to local doubling. In addition to this there was some vascularization of the epithelium by vessels budding from the choroidal vasculature. The vascular atrophy clinically observed in vivo, had its histological correlate in the form of a few tortuous capillaries within the choroid, and of a reduced and abnormally distributed retinal capillary net. The only obvious change within the inner layers was the presence of distinctly hypertrophic Muller cell processes. The effect appears to represent a reaction of the Muller cell processes, which become more readily stainable and more electron dense, rather than actual glial proliferation.

\section{Survival and Growth of the Transplants}

From seven to 10 days post-transplant (PTD), fundus examination showed transplants growing as irregular masses, in contact with the host retina. By examining the bisected eyes under the stereo microscope, it was possible to locate the transplant in approximately two-thirds of the transplanted eyes.

Although the host eyes invariably exhibited profound pathological changes related to the noxious effects of light exposure, the transplants grew well, leading to the development of clusters of photoreceptor cells embedded into the host retina. Depending on the experimental group the study of microscopic sections of implanted eyes revealed that up to $80 \%$ had transplanted tissue located preferentially at the point of injection (Fig. 6). Fluorescence microscopy (Fig. 7) showed these clusters to be formed by fluorescently labelled cells developing in intimate contact with the unlabelled host retina. In every case, clusters of rod cells developed with their somata forming an irregular outer nuclear layer closely integrated with the host (Fig. 6). The rod cells were often grouped in rosettes. Rod cell inner segments developed consistently. As a uniform feature they possessed a basal body with a cilium emerging from it. The outer segments that formed in many of the rosettes tended to be defective, consisting of collections of irregular cisternea. Both conventional and ribbon synapses were present in large numbers within the patches of plexiform layer which developed around the clusters of transplanted photoreceptors (Figs. 8a and b). Integration of the transplant with the host retina was revealed by physical continuity, common vascularization, and consistent lack of glial barriers around the transplants.

\section{Changes in the Host Retina}

Histological changes directly or indirectly attributable to the presence of the transplant occurred in the retinas of some of the hosts. These changes varied, however, in their location and extent. Some of the observed changes were the direct result of the surgical intervention. The most obvious was the minute scar formed at the injection site. The presence of small subretinal hemorrhages, and of some pigment-laden macrophages in the subretinal space adjacent to the penetration site was in all likelihood related to the microsurgical trauma. The presence of some free red cells and active macrophages in the vitreous may have also represented a response to

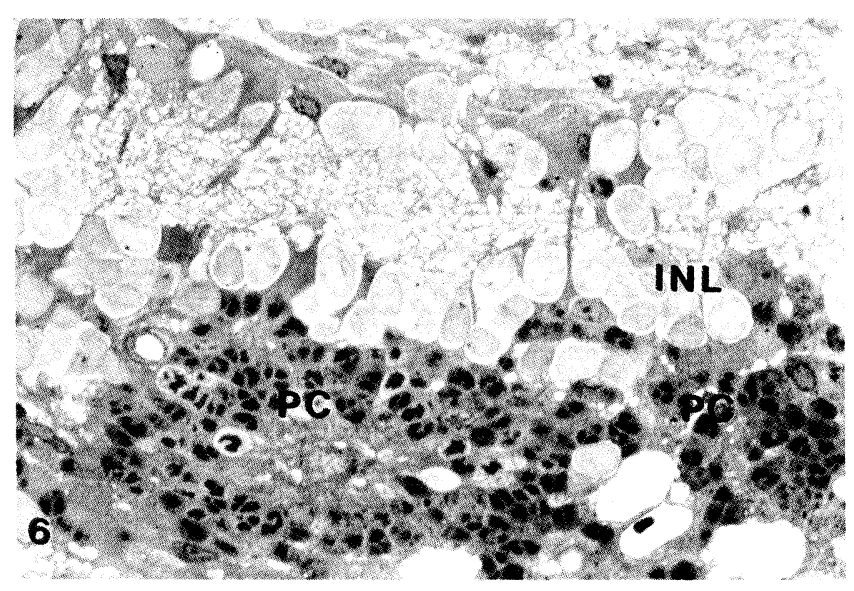

Fig. 6: Cluster of photoreceptor cells (PC) within a lightirradiated retina that have received a transplant of dissociated retinal cells $\mathbf{3 0}$ days before sacrifice. (INL) indicates the host inner nuclear layer. Two $\mu \mathrm{m}$-thick plastic section. Magnification $500 \mathrm{x}$.

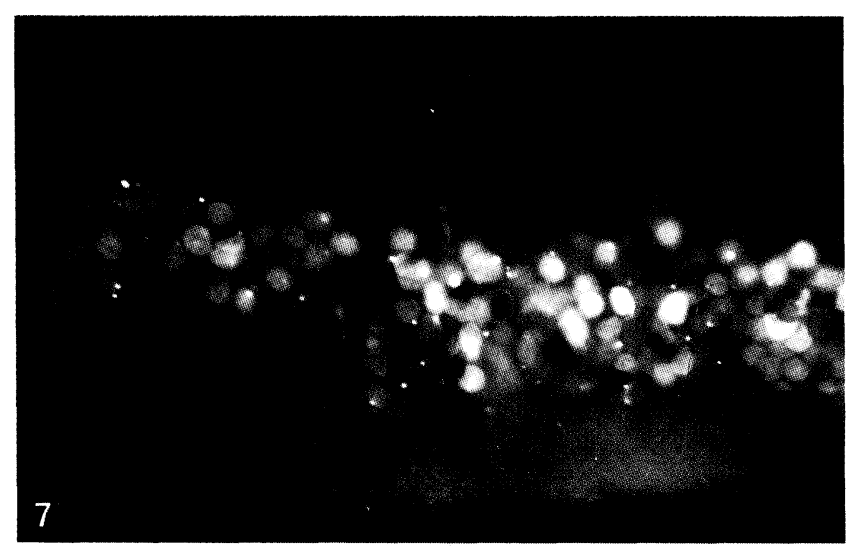

Fig. 7: Fast-blue labelled cells in the retina of a light-irradiated host sacrificed 30 days post-transplantation. The cells have clustered into a layer-like formation. Fluorescence microphotograph of a cryostat section. $500 \mathrm{x}$. 


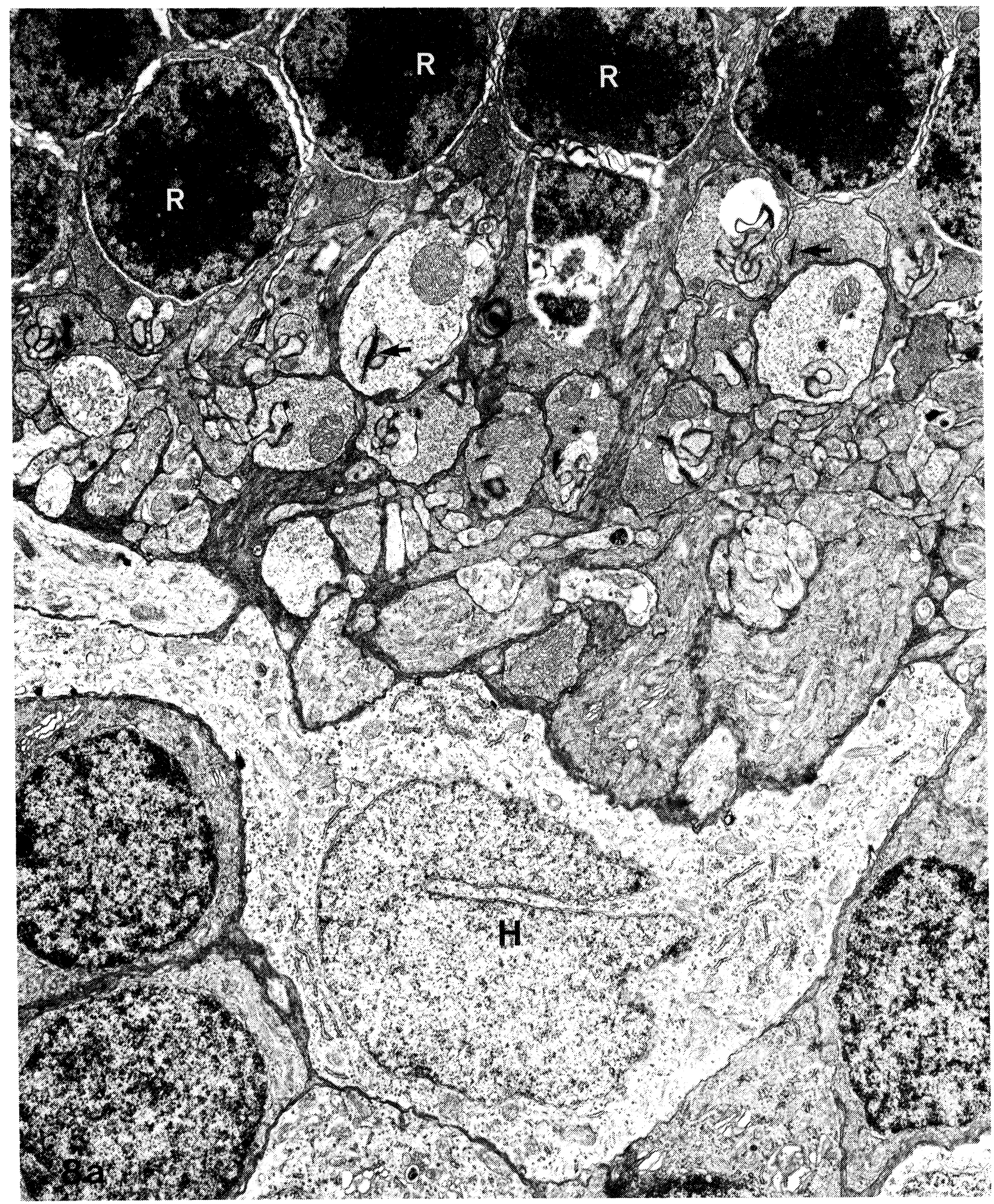

Fig. 8a: Electron micrograph showing an area of host retina repopulated by rod cells (R) 30 days after transplantation. Synaptic contacts can be seen in the neighborhood of transplanted rod cells, either within small patches of plexiform layer or intermingled with rod cell somas. Arrows indicate some of the ribbons present within photoreceptor terminals. $(H)$ indicates a horizontal cell. Magnification $8,900 \mathrm{x}$. 


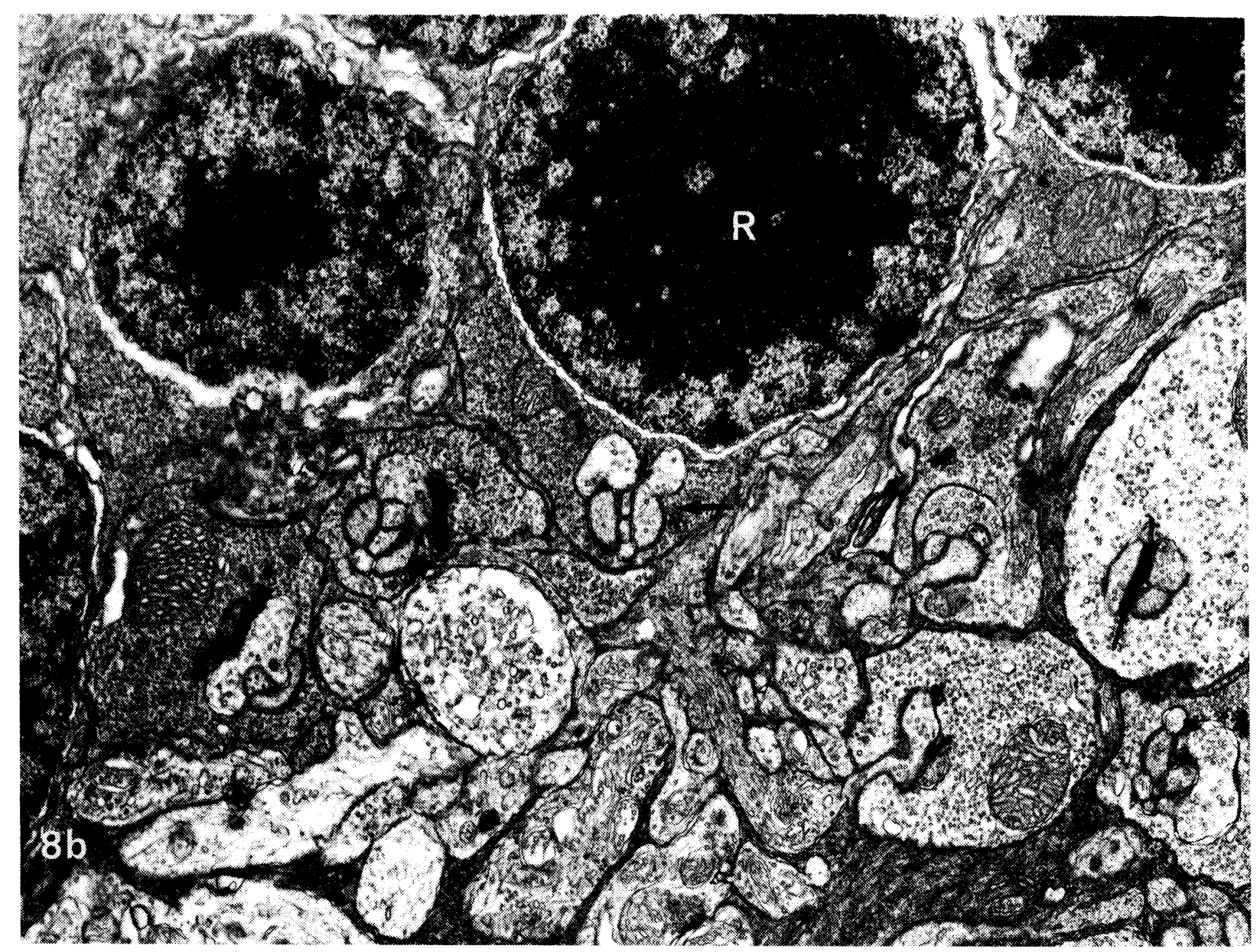

Fig. 8b: An enlarged sector of the previous figure shows details of the synaptic contacts within the transplant. One of these contacts (arrow) takes place inside the cytoplasm of a rod cell (R), which has been penetrated by post-synaptic elements. Magnification $15,000 \mathrm{x}$.

the injection trauma, otherwise the transplantation of dissociated cells elicits no reaction in the host eye.

\section{DISCUSSION}

\section{Light-Induced Damage}

The deleterious effects on the retinal photoreceptor cells caused by continuous exposure to low levels of visible radiation have been well known since the classic studies of Noell et al. /18/. Our experimental protocol, based on those observations, involved prolonged exposure to moderate light intensities. The result was the induction of the so-called type II light damage /19/, characterized by diffuse photoreceptor loss with general preservation of the retinal pigment epithelial cells. Type I damage, triggered by shorter exposure to high light intensities, is characterized by generalized loss of both photoreceptors and retinal pigment epithelial cells. Photic lesions resulting from chronic exposure to moderate illumination intensities have been described previously / $13,20 /$. Some differences exist in the results obtained by different laboratories studying late-stage phototoxic damage, these differences may reflect differences in experimental protocols, genetic backgrounds, etc. /16,21/.

Besides photoreceptor cell decimation, a marked reduction in the retino-choroidal vascular bed was observed in our material. This phenomenon is in all likelihood, secondary to the extended destruction of the neuronal population. In fact, vascular correlates of photoreceptor decimation have been reported by Bellhorn and his associates $/ 3 /$. The vascular atrophy may be induced by the lowered metabolic requirements of a retina greatly reduced in total mass, rather than by direct actinic action. The occurrence of a comparable vascular attenuation in genetically induced retinal degenerations $/ 5 /$ lends support to the notion that reduced metabolic demands trigger vascular regression. 


\section{Graft Related Issues}

Our previous studies have shown the receptivity of the adult rat eye to transplants of embryonic and perinatal retinas even in transplants made across strains $19,10,11 /$. As a logical second step we wished to determine if eyes affected by a near total destruction of the photoreceptor cell population and a severely compromised vascular supply, would also be able to incorporate transplanted cells and to support their growth and differentiation. The foregoing observations gave positive answers to these questions. They demonstrate that normal perinatal retinal cells transplanted into a retina are able to differentiate to a considerable degree, and to supply the host with new photoreceptor cells. On the other hand, the laminar organization attained by these transplants was less regular than that seen in other forms of intraocular transplants $/ 4,10 /$. A possible explanation for these findings is that the differentiation into photoreceptors may be achieved by th e immature cells following internal clues, while the laminar arrangement may depend to a large extent on the timely action of extrinsic factors which are absent from the light damaged host retina $/ 1 /$.

In the presence of successful neural grafting of any kind, two essential issues need to be discussed; they are: 1) the unambiguous identification of the transplanted cells within the host tissue, and 2) the extent and nature of the relationships of the transplanted cells with the host tissue. In the case of retinal transplants into eyes being affected by late stage phototoxic retinopathy, the first question is easily answered. As a result of the stringent experimental protocol devised for this experiment, the central and equatorial regions of the hosts lose practically every single receptor cell. In the far retinal periphery only a few atrophic photoreceptor cells remained. The photic damage to the periphery is less severe than that occurring in the equatorial and central regions, but this increased resistance is counterbalanced in the Fisher 344 rat by the spontaneous tendency to degenerate expressed by the peripheral retina $/ 14 /$. The combined effects of these two factors is that our hosts appear to have retinas further depopulated of photoreceptor cells than most of the light irradiated animals previously reported in the literature. Thus the presence of clusters formed by several hundred photoreceptor cells unmistakably identified the graft. Comparable clusters of photoreceptor cells have never been observed in any of the more than one hundred phototoxic retinitis-affected eyes we have studied in conjunction with this and other studies. As described in the results section, the presence of fluorescent label within the cells confirms the notion that these clusters are indeed formed by donor cells /12/. Finally, electron microscopical observations show an important difference between the ultrastructure of the few survival rod cells seen in the non-transplanted, phototoxic retinitis-affected hosts and those present within the transplanted clusters. The latter are distinguished by their tendency to develop inner and outer segments. Although these structures do not develop normally within the transplants, their mere presence sets the donor cells aside from those seen in irradiated by non-transplanted animals, or in areas outside the transplant region. In either of those cases, the few remaining rod cells are consistently deprived of both inner and outer segments. Actually the loss of outer and inner segments is an early event in the development of phototoxic retinitis /13/.

The second and more involved question is that of the relationship between the transplanted photoreceptors and the host retina. In this regard the use of cell suspensions $/ 2 /$ offers definite advantages over the transplantation of retinal strips /12/. The electron microscope shows areas of plexiform layers around the clusters of transplanted photoreceptors. Numerous synaptic contacts, including ribbon synapses are found within these regions. Some of these contacts are abnormal, for example those occurring as invaginations within the photoreceptor cell soma, instead of being on a pedicle projecting from the cell body. Nonetheless, these cells retain enough of their typical morphology so as to be recognized as outer plexiform layer synaptic contacts. This evidence indicates that the transplanted photoreceptors are capable of linking synaptically to other cells. Two possibilities, which are not mutually exclusive, do exist regarding the origin of the synaptic partners to the transplanted photoreceptors. The photoreceptors may be in synaptic contact with the host inner nuclear layer neurons, or with co-transplanted cells, or both. An unequivocal answer to this question will require differential labelling of the host cells with an electron microscopically identifiable tag. Efforts in this direction are currently underway.

The development of some outer segments in the absence of contacts with the RPE parallels similar observations in different experimental situations. LaVail and Hild /15/ noticed that rod outer segments may differentiate to a considerable degree in vitro in the absence of RPE. Similar differentiation of outer segments has been known to occur in intracranially transplanted neuroretinas $/ 17 /$.

Three basic requirements have to be met for any 
degree of anatomical repair to take place in a damaged neural system: first, replacement of cells that have died with similar cells or their precursors; second, differentiation of these cells and development of synaptic competency; third, an activation of the plastic properties of the host's remaining neurons has to occur for them to develop synaptic partnership with the transplanted cells. It is not unreasonable to expect that some degree of functional recovery may occur if these requirements are met by enough cells within the damaged structure. The present results suggest that the model exists for testing the functional correlates of retinal transplantation in damaged adult retinas.

\section{REFERENCES}

1. Adler R, Hatlee M. Plasticity and differentiation of embryonic retinal cells af ter terminal mitosis. Science 1989; 243: 391-393.

2. Bjorklund A, Stenevi U, Schmidt RH, Dunnet SB, Gage FH. Intracerebral grafting of neuronal cell suspensions. I. Introduction and general methods of preparation. Acta Physiol Scand Suppl 1983; 522: 1-7.

3. Bellhorn RW, Burns MS, Benjamin JV. Retinal vessel abnormalities of phototoxic retinopathy in rats. Invest Ophthalmol Vis Sci 1980; 19: 584-595.

4. Blair JR, Turner JE. Optimum conditions for successful transplantation of immature rat retina to the lesioned adultretina. Dev Brain Res 1987; 36: 257-270.

5. Blanks JC, Johnson LV. Vascular atrophy in the retinaldegenerative rd mouse. J Comp Neurol 1986; 254: 543-553.

6. Dalton AJ. A chrome-osmium fixative forelectron microscopy. Anat Rec 1955; 121: 281.

7. del Cerro M, Cogen MJ, del Cerro C. Stevenel Blue, an excellent stain for opticalmicroscopy study of plastic embedded tissues. Microscop Acta 1980a; 83: 117-121.

8. del Cerro M, Standler MN, del Cerro C. high resolution optical microscopy of animal tissues by the use of sub-micrometer thick sections and a new stain. Microscop Acta $1980 \mathrm{~b} ; 83: 217-220$.
9. del Cerro M, Gash DM, Rao GN, Notter MF, Wiegand SJ, Gupta M. Intraocular retinal transplants. Invest Ophthalmol Vis Sci 1984; 25: 62 (abstr.).

10. del Cerro M, Gash DM, Rao GN, Notter MF, Wiegand SJ, Gupta M. Intraocular retinal transplants. Invest Ophthalmol Vis Sci 1985; 26: 1182-1185.

11. del Cerro M, Gash DM, Rao GN, Notter MF, Wiegand SJ, Sathi S, del Cerro C. Retinal transplants into the anterior chamber of the rat eye. Neuroscience $1987 \mathrm{c} ; 21: 707-724$.

12. del Cerro M, Notter MF, Wiegand SJ, Jiang LQ, del Cerro C. Intraretinal transplantation of fluorescently labeled retinal cell suspensions. Neurosci Lett 1988a; 92: 21-26.

13. Kwabara T, Gorn RA. Retinal damage by visible light. Arch Ophtlamol 1968; 79: 69-78.

14. Lai YL, Jacoby RO, Jonas AM. Age-related and light-associated retinal changes in Fisher rats. Invest Ophthalmol Vis Sci 1978; 17: 634-638.

15. LaVail MM, Hild W. Histotypic organization of the rat retina in vitro. $Z$ Zellforsch 1971; 114: 557-579.

16. LaVail MM, Gorrin GM, Repaci MA, Yasumura D. Lightinduced retinal degeneration in albino rats and mice: strain and species differencies. In: Hollyfield JG, Anderson RE, LaVail MM, eds., Degeneration Retinal Disorders: Clinical and Laboratory Investiations. New York: A.R. Lis, 1987; 439-454.

17. Lund RD, Rao K, Hankin MH, Kunz HW, Gill TJ Transplantation of retina and visual cortex to rat brains of different ages. Maturation, Connections, patterns, and immunological consequences. Ann NY Acad Sci 1987; 495: 227-240.

18. Noell WK, Walker VS, Kang BS, Berman S. Retinal damage by light in rats. Invest Ophthalmol Vis Sci 1966; 5: 450-473.

19. Noell WK. Possible mechanisms of photoreceptor ddamage by light in mammalian eyes. Vision Res 1980; 20: 1163.

20. Parnavelas JG, Globus A. The damaging effects of continuous illumination on the morphology of the rat retina. Exp Neurol 1976; 51: 171-187.

21. Penn JS, Howard AG, Williams ThP. Light damage as a function of "light history" in the albino rat. In: Retinal Degeneration: Experimental and Clinical Studies. New York: Alan R. Liss, 1985; 439-447.

22. Venable JH, Cogeshall R. A simplifed lead citrate stain for use in electron microscopy. J Cell Biol 1965; 25: 407-408. 

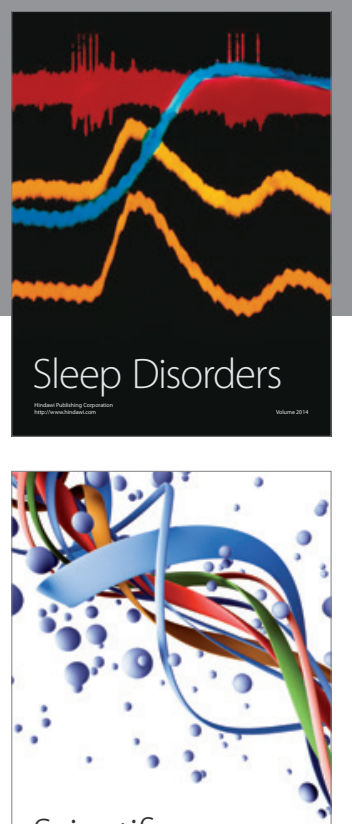

Scientifica
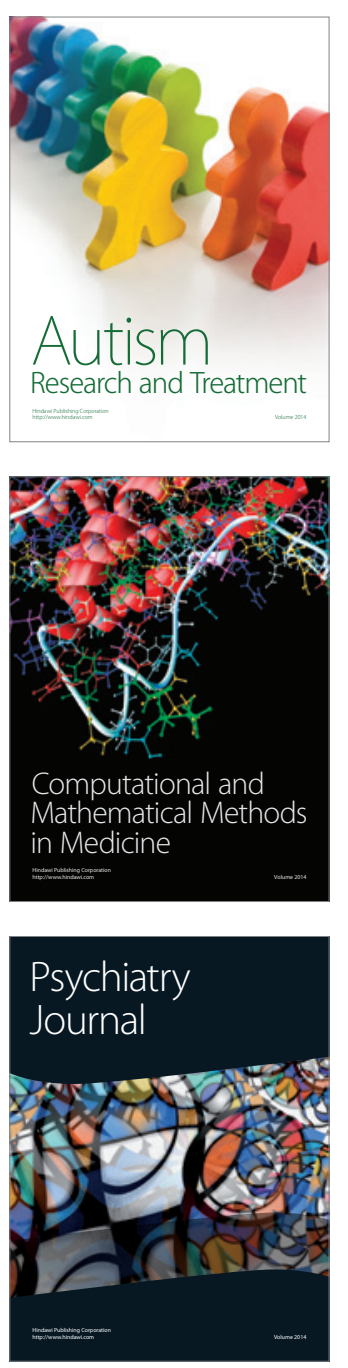
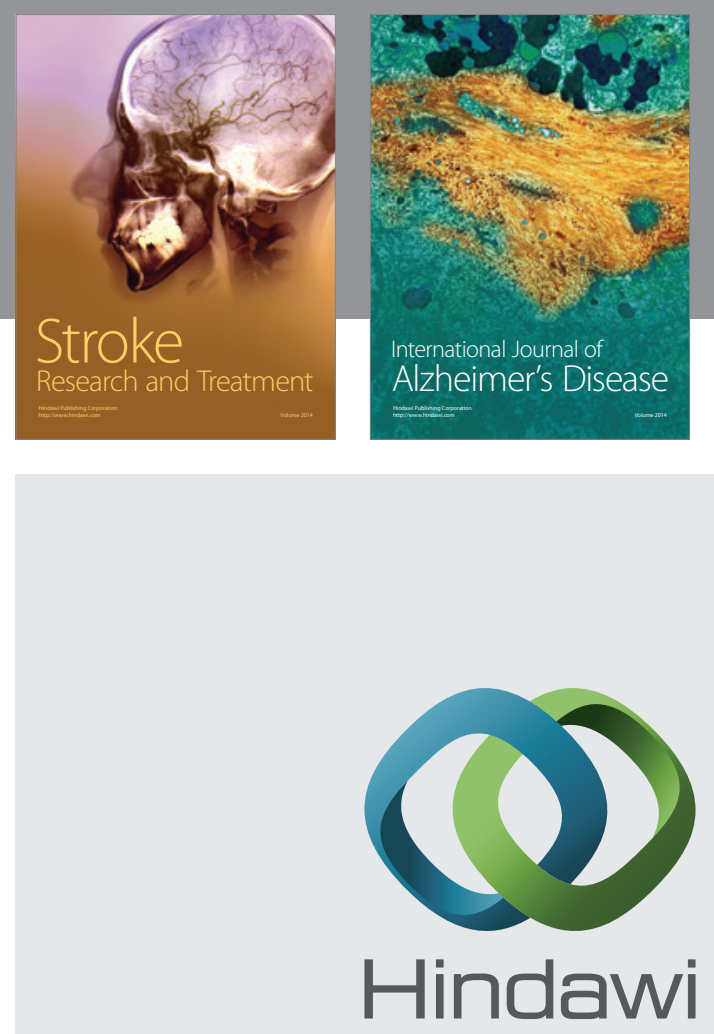

Submit your manuscripts at

http://www.hindawi.com
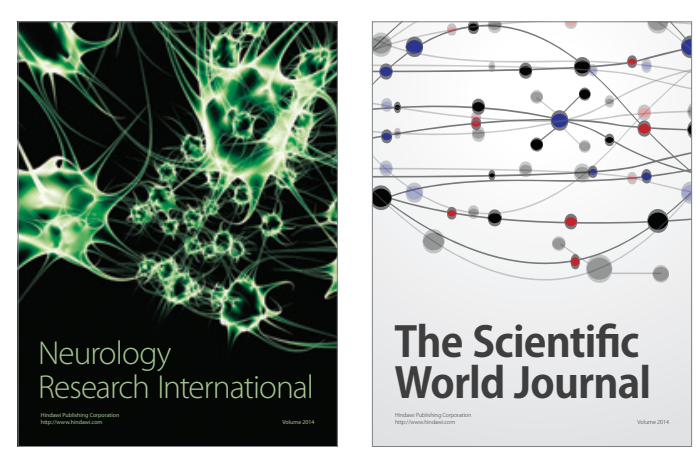

The Scientific World Journal

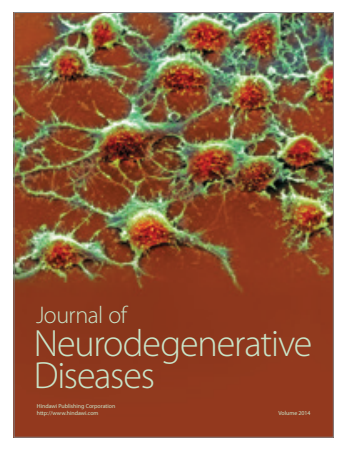

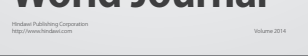

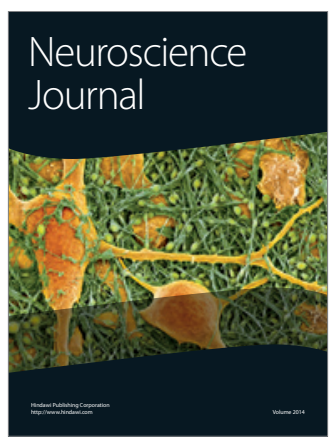

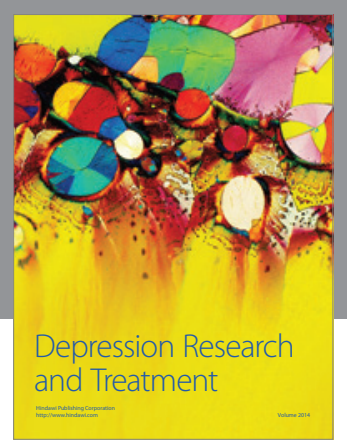
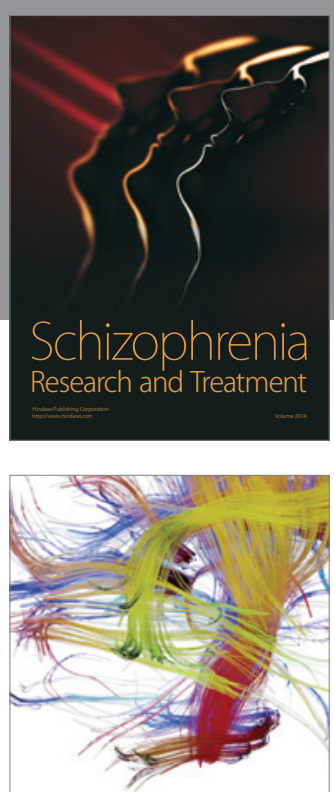

Brain Science

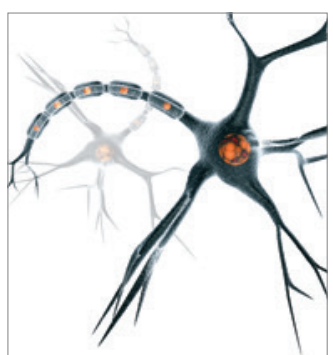

Neural Plasticity
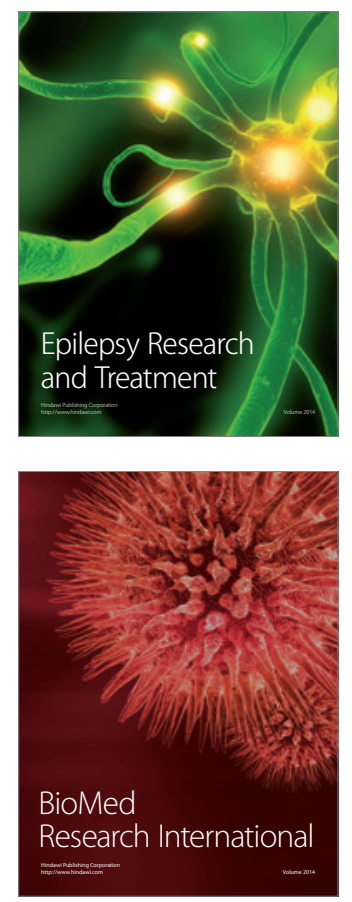

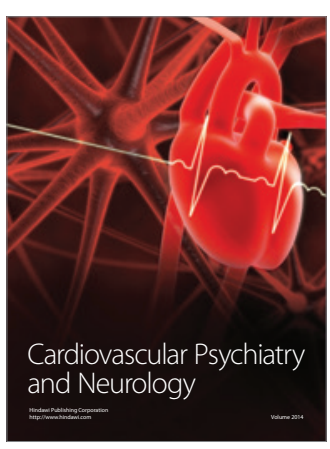

Parkinson's

Disease
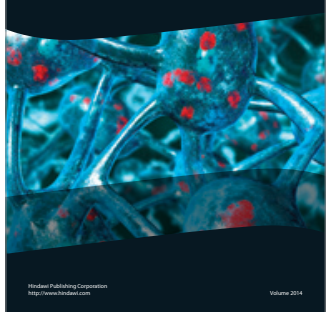